\title{
GROUP SPARSITY BASED WIDEBAND DOA ESTIMATION FOR CO-PRIME ARRAYS
}

\author{
Qing Shen*†, Wei Liu ${ }^{\dagger}$, Wei Cui*, Siliang Wu*, Yimin D. Zhang ${ }^{\ddagger}$, Moeness G. Amin ${ }^{\ddagger}$ \\ ${ }^{*}$ School of Information and Electronics, Beijing Institute of Technology \\ Beijing, 100081, China \\ ${ }^{\dagger}$ Department of Electronic and Electrical Engineering, University of Sheffield \\ Sheffield, S1 3JD, United Kingdom \\ $\ddagger$ Center for Advanced Communications, Villanova University \\ Villanova, PA 19085, USA
}

\begin{abstract}
A novel wideband direction-of-arrival (DOA) estimation method is proposed for co-prime arrays. After decomposing the wideband signals into different frequencies/subbands through a discrete Fourier transform or, more generally, a filter bank system, the increased degrees of freedom provided by co-prime arrays are fully exploited with a group sparsity based signal reconstruction method. Simulation results show that this novel method can distinguish much more sources than the number of physical sensors. Compared with the existing narrowband DOA estimation method for co-prime arrays, the proposed wideband method achieves a significant performance improvement.
\end{abstract}

\section{INTRODUCTION}

To avoid spatial aliasing, the minimum spacing between adjacent sensors in a uniform linear array (ULA) should be less than $\lambda_{\min } / 2$, where $\lambda_{\min }$ is the minimum wavelength within the frequency band of interest [1], [2]. This can be problematic when considering arrays with a large aperture size, due to the cost associated with the number of sensors. In the past, sparse arrays have been proposed to avoid this problem [3][6], where their non-uniform nature can avoid grating lobes while allowing adjacent sensor spacings to be greater than $\lambda_{\min } / 2$. Minimum redundant linear array (MRLA) is a typical example of sparse arrays [7]. However, there is no systematic approach for finding an MRLA when the number of array sensors is large.

Recently, a new class of sparse arrays called co-prime array was proposed [8], [9]. Assume $M$ and $N$ are a pair of co-prime positive integers. Then, a co-prime array can be constructed by two sub-arrays, one with $N$ sensors and one with $M$ sensors. The adjacent sensor spacing for the first sub-array is $M d$, while it is $N d$ for the second one, where $d$ is the unit spacing. The entire co-prime array only has $M+N-1$ sensors, but the difference co-array can provide more than $M N$ degrees of freedom. The increased degrees of freedom

The work of Y. D. Zhang and M. G. Amin was supported in part by the Office of Naval Research under Grant N00014-13-1-0061. can be exploited in both beamforming and direction of arrival (DOA) estimation [9]-[12]. In [9], a virtual array of a larger aperture is generated from the co-prime array by vectorizing the autocorrelation matrix. Then, rank restoring method based upon spatial smoothing is utilized for narrowband DOA estimation. Sparsity-based signal recovery method is proposed under the condition of imperfect correlation matrix in [10]. In [12], a sparse signal recovery method based on compressive sensing is used for narrowband DOA estimation, exploiting a ULA with two co-prime frequencies. All the methods above are designed for narrowband waveforms. The problem of how to make full use of these increased degrees of freedom for a wideband co-prime array has not been addressed yet.

Many wideband DOA estimation methods have been proposed in the past, such as the incoherent signal subspace method (ISSM) [13], the coherent signal subspace method (CSSM) [14], and the recently proposed test of orthogonality of projected subspaces (TOPS) method [15]. In particular, a series of DOA estimation methods based on the sparse signal recovery approach were developed in [16], [17]. In [18], a subband information fusion (SIF) method based on the concept of group sparsity is introduced to jointly explore the information in all subbands.

In this paper, a method for wideband DOA estimation for co-prime arrays based on group sparsity is proposed, which can be considered as a further extension of the method in [11]. At first, the wideband signals received by the array are decomposed into different frequencies/subbands by a discrete Fourier transform (DFT) or, more generally, a filter bank system. Virtual arrays are then formed by vectorizing the auto-correlation matrix in each subband. As the equivalent signal vector of each virtual array is a column vector consisting of all impinging signal powers, sparsity-based signal recovery method could be used for DOA estimation at each subband. In order to jointly exploit the information provided by all subbands, the groupsparsity based signal reconstruction method is employed in this paper for wideband DOA estimation. Simulation results 


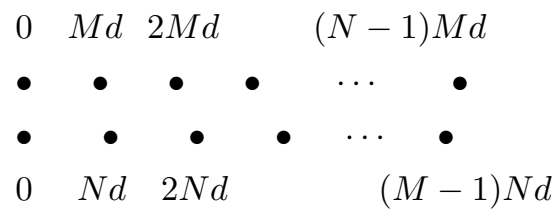

Fig. 1. Structure of a general co-prime array.

show that a significantly improved performance is achieved by our proposed method.

This paper is organized as follows. The wideband signal model for co-prime arrays is presented in Section II. The proposed wideband DOA estimation method is introduced in Section III. Simulation results are provided in Section IV, and conclusions are drawn in Section V.

\section{Wideband Signal Model With Co-Prime ARrays}

A co-prime array consists of two uniform linear sub-arrays, as shown in Fig. 1. $M$ and $N$ are co-prime and $M<N$ is assumed. The first sub-array has $N$ sensors with a spacing of $M d$, and the second sub-array has $M$ sensors with a spacing of $N d$, where $d \leq \lambda_{\min } / 2$.

The zeroth positions of the two subarrays share the same sensor and in total there are $M+N-1$ sensors. The set of sensor positions for each sub-array can be expressed as $S_{1}$ and $S_{2}$, where the zeroth sensor is removed from $S_{2}$ for convenience of formulation at the next stage:

$$
\begin{aligned}
& S_{1}=\{M n d, 0 \leq n \leq N-1, n \in Z\}, \\
& S_{2}=\{N m d, 1 \leq m \leq M-1, m \in Z\} .
\end{aligned}
$$

Assume that there are $K$ uncorrelated wideband signals $s_{k}(t)$ with the same bandwidth impinging from incident angles $\theta_{k}, k=1,2, \ldots, K$, respectively, where $\theta_{k}$ is measured from the broadside of the array. Then, the signals observed from the two sub-arrays can be expressed as:

$$
\begin{aligned}
x_{1, n}(t) & =\sum_{k=1}^{K} s_{k}\left[t-\tau_{1, n}\left(\theta_{k}\right)\right]+\bar{n}_{1, n}(t), \\
x_{2, m}(t) & =\sum_{k=1}^{K} s_{k}\left[t-\tau_{2, m}\left(\theta_{k}\right)\right]+\bar{n}_{2, m}(t),
\end{aligned}
$$

where $0 \leq n \leq N-1$ and $1 \leq m \leq M-1$. Take the zeroth position of the co-prime array as the reference. Then, $\tau_{1, n}\left(\theta_{k}\right)$ and $\tau_{2, m}\left(\theta_{k}\right)$ represent the time delay of the $k$-th impinging signal with the incident angle $\theta_{k}$ arriving at the $n$-th sensor of the first sub-array and the $m$-th sensor of the second subarray, respectively. $\bar{n}_{1, n}(t)$ and $\bar{n}_{2, m}(t)$ are noise observed at the corresponding sensors.

With a sampling frequency $f_{s}$, the discrete version of the two sets of sub-array signals can be expressed as

$$
\begin{aligned}
& \mathbf{x}_{\mathbf{1}}[i]=\left[x_{1,0}[i], x_{1,1}[i], \ldots, x_{1, N-1}[i]\right]^{T}, \\
& \mathbf{x}_{\mathbf{2}}[i]=\left[x_{2,1}[i], x_{2,2}[i], \ldots, x_{2, M-1}[i]\right]^{T},
\end{aligned}
$$

where \{\}$^{T}$ denotes the transpose operation.

For each received sensor signal, every $L$ samples are divided into one group, and an $L$-point DFT is applied. There is no overlap between adjacent DFTs. Then, the $l$-th frequency bin/subband samples of the $p$-th group for each sub-array can be grouped into one vector as follows

$$
\begin{aligned}
& \mathbf{X}_{1}[l, p]=\left[X_{1,0}[l, p], X_{1,1}[l, p], \ldots, X_{1, N-1}[l, p]\right]^{T}, \\
& \mathbf{X}_{\mathbf{2}}[l, p]=\left[X_{2,1}[l, p], X_{2,2}[l, p], \ldots, X_{2, M-1}[l, p]\right]^{T},
\end{aligned}
$$

where

$$
\begin{aligned}
& X_{1, n}[l, p]=\sum_{i=0}^{L-1} x_{1, n}[L \cdot(p-1)+i] \cdot e^{-j \frac{2 \pi}{L} i l}, \\
& X_{2, m}[l, p]=\sum_{i=0}^{L-1} x_{2, m}[L \cdot(p-1)+i] \cdot e^{-j \frac{2 \pi}{L} i l},
\end{aligned}
$$

with $p=1, \ldots, P$, and $0 \leq l \leq L-1$

Define $S_{k}[l, p], \bar{N}_{1, n}[l, p]$, and $\bar{N}_{2, m}[l, p]$ as the DFT of the $p$-th group discrete-time impinging signals $s_{k}[i]$, discretetime noises at sensors of the two sub-arrays $\bar{n}_{1, n}[i]$ and $\bar{n}_{2, m}[i]$, respectively. $\mathbf{S}[l, p]=\left[S_{1}[l, p], \ldots, S_{K}[l, p]\right]^{T}$ is a column vector holding signals at the $l$-th frequency bin, and $\overline{\mathbf{N}}_{1}[l, p]=\left[\bar{N}_{1,0}[l, p], \ldots, \bar{N}_{1, N-1}[l, p]\right]^{T}$ and $\overline{\mathbf{N}}_{2}[l, p]=$ $\left[\bar{N}_{2,1}[l, p], \ldots, \bar{N}_{2, M-1}[l, p]\right]^{T}$ are the corresponding column noise vectors at the two sub-arrays. Then, the output signal model at the DFT domain can be expressed as

$$
\begin{aligned}
& \mathbf{X}_{1}[l, p]=\mathbf{A}_{1}(l, \boldsymbol{\theta}) \mathbf{S}[l, p]+\overline{\mathbf{N}}_{1}[l, p], \\
& \mathbf{X}_{2}[l, p]=\mathbf{A}_{2}(l, \boldsymbol{\theta}) \mathbf{S}[l, p]+\overline{\mathbf{N}}_{2}[l, p],
\end{aligned}
$$

where $\mathbf{A}_{1}(l, \boldsymbol{\theta})=\left[\mathbf{a}_{1}\left(l, \theta_{1}\right), \ldots, \mathbf{a}_{1}\left(l, \theta_{K}\right)\right]$ and $\mathbf{A}_{2}(l, \boldsymbol{\theta})=$ $\left[\mathbf{a}_{2}\left(l, \theta_{1}\right), \ldots, \mathbf{a}_{2}\left(l, \theta_{K}\right)\right]$ are the steering matrices at frequency $f_{l}$ corresponding to the $l$-th frequency bin. The column vectors $\mathbf{a}_{\mathbf{1}}\left(l, \theta_{k}\right)$ and $\mathbf{a}_{\mathbf{2}}\left(l, \theta_{k}\right)$ are the steering vectors at frequency $f_{l}$ and angle $\theta_{k}$.

\section{Proposed Wideband DOA Estimation Method BASED ON GROUP SPARSITY}

\section{A. Virtual array generation}

Denote $\mathbf{X}[l, p]=\left[\mathbf{X}_{1}^{T}[l, p], \mathbf{X}_{2}^{T}[l, p]\right]^{T}$. Then, the autocorrelation matrix of the observed array vector $\mathbf{X}[l, p]$ is

$$
\begin{aligned}
\mathbf{R}_{\mathbf{x} \mathbf{x}}[l] & =E\left\{\mathbf{X}[l, p] \cdot \mathbf{X}^{H}[l, p]\right\} \\
& =\sum_{k=1}^{K} \sigma_{k}^{2}[l] \mathbf{a}\left(l, \theta_{k}\right) \mathbf{a}^{H}\left(l, \theta_{k}\right)+\sigma_{n}^{2}[l] \mathbf{I}_{\mathbf{M}+\mathbf{N}-\mathbf{1}},
\end{aligned}
$$

where \{\}$^{H}$ denotes Hermitian transpose, $E\{\}$ is the statistical expectation operator, $\mathbf{a}\left(l, \theta_{k}\right)=\left[\mathbf{a}_{1}^{T}\left(l, \theta_{k}\right), \mathbf{a}_{2}^{T}\left(l, \theta_{k}\right)\right]^{T}$ and $\mathbf{I}_{\mathbf{M}+\mathbf{N}-\mathbf{1}}$ is the $(M+N-1) \times(M+N-1)$ identity matrix. $\sigma_{k}^{2}[l]$ represents the power of the $k$-th impinging signal at the $l$-th frequency bin, and $\sigma_{n}^{2}[l]$ is the corresponding noise power. In practice, $\mathbf{R}_{\mathbf{x x}}[l]$ can be estimated by

$$
\mathbf{R}_{\mathbf{x} \mathbf{x}}[l] \approx \widehat{\mathbf{R}}_{\mathbf{x} \mathbf{x}}[l]=\frac{1}{P} \sum_{p=1}^{P} \mathbf{X}[l, p] \cdot \mathbf{X}^{H}[l, p],
$$


where $P$ is the number of signal blocks for DFT.

Vectorizing $\mathbf{R}_{\mathbf{x x}}[l]$ yields

$$
\mathbf{z}[l]=\operatorname{vec}\left\{\mathbf{R}_{\mathbf{x x}}[l]\right\}=\widetilde{\mathbf{A}}[l] \widetilde{\mathbf{s}}[l]+\sigma_{n}^{2}[l] \widetilde{\mathbf{I}}_{\mathbf{M}+\mathbf{N}-\mathbf{1}},
$$

where $\widetilde{\mathbf{A}}[l]=\left[\widetilde{\mathbf{a}}\left(l, \theta_{1}\right), \ldots, \widetilde{\mathbf{a}}\left(l, \theta_{K}\right)\right]$ with $\widetilde{\mathbf{a}}\left(l, \theta_{k}\right)=$ $\mathbf{a}^{*}\left(l, \theta_{k}\right) \otimes \mathbf{a}\left(l, \theta_{k}\right)(\otimes$ is the Kronecker product), and $\widetilde{\mathbf{s}}[l]=$ $\left[\sigma_{1}^{2}[l], \ldots, \sigma_{K}^{2}[l]\right]^{T} . \widetilde{\mathbf{I}}_{\mathbf{M}+\mathbf{N}-\mathbf{1}}$ is a $(M+N-1)^{2} \times 1$ column vector obtained by vectorizing the identity matrix $\mathbf{I}_{\mathbf{M}+\mathbf{N}-\mathbf{1}}$.

Eq. (9) characterises a virtual array, with $\widetilde{\mathbf{A}}[l]$ as its steering matrix and $\widetilde{\mathbf{s}}[l]$ as its equivalent impinging signal vector. Moreover, (9) can be modified into

$$
\mathbf{z}[l]=\widetilde{\mathbf{A}}[l] \widetilde{\mathbf{s}}[l]+\sigma_{n}^{2}[l] \widetilde{\mathbf{I}}_{\mathbf{M}+\mathbf{N}-\mathbf{1}}=\widetilde{\mathbf{A}}^{\circ}[l] \widetilde{\mathbf{s}}^{\circ}[l],
$$

where $\widetilde{\mathbf{A}}^{\circ}[l]=\left[\widetilde{\mathbf{A}}[l], \widetilde{\mathbf{I}}_{\mathbf{M}+\mathbf{N}-1}\right]$ and $\widetilde{\mathbf{s}}^{\circ}[l]=\left[\widetilde{\mathbf{s}}^{T}[l], \sigma_{n}^{2}[l]\right]^{T}$.

\section{B. DOA estimation based on single frequency}

For the $l$-th frequency, with a search grid of $K_{g}$ potential incident angles $\theta_{g, 1}, \ldots, \theta_{g, K_{g}}$, the steering matrix is generated by $\widetilde{\mathbf{A}}_{\mathbf{g}}[l]=\left[\widetilde{\mathbf{a}}\left(l, \theta_{g, 1}\right), \ldots, \widetilde{\mathbf{a}}\left(l, \theta_{g, K_{g}}\right)\right]$. Construct a column vector $\widetilde{\mathbf{S}}_{\mathbf{g}}[l]$ consisting of $K_{g}$ elements, with each of them representing a potential source signal at the corresponding incident angle. Denote

$$
\begin{aligned}
\widetilde{\mathbf{A}}_{\mathbf{g}}^{\circ}[l] & =\left[\widetilde{\mathbf{A}}_{\mathbf{g}}[l], \widetilde{\mathbf{I}}_{\mathbf{M}+\mathbf{N}-\mathbf{1}}\right], \\
\widetilde{\mathbf{s}}_{\mathbf{g}}^{\circ}[l] & =\left[\widetilde{\mathbf{s}}_{\mathbf{g}}[l], \sigma_{n}^{2}[l]\right]^{T} .
\end{aligned}
$$

The last element $\sigma_{n}^{2}[l]$ in $\widetilde{\mathbf{s}}_{\mathbf{g}}^{\circ}[l]$ can also be considered as a variable because the noise power is unknown. A compressive sensing based DOA estimation method for narrowband coprime array was proposed in [11], and it can be applied to a single frequency in the wideband case directly. The formulation for the $l$-th frequency $f_{l}$ is shown in the following:

$$
\begin{aligned}
\min & \left\|\widetilde{\mathbf{s}}_{\mathbf{g}}^{\circ}[l]\right\|_{1} \\
\text { subject to } & \left\|\mathbf{z}[l]-\widetilde{\mathbf{A}}_{\mathbf{g}}^{\circ}[l] \widetilde{\mathbf{s}}_{\mathbf{g}}^{\circ}[l]\right\|_{2} \leq \varepsilon,
\end{aligned}
$$

where $\varepsilon$ is the allowable error bound, $\|\cdot\|_{1}$ is the $l_{1}$ norm and $\|\cdot\|_{2}$ the $l_{2}$ norm.

\section{Wideband extension based on group sparsity}

We could apply the algorithm in (12) to the frequency range of interest one by one and then average the results to give the final estimation. A more effective approach, however, is to estimate the DOA of the impinging signals across the frequency range of interest simultaneously based on the group sparsity concept, i.e. the DOAs corresponding to different frequencies share the same spatial support, although they in general have varying power values.

Assume that the frequency range or bandwidth of interest covers $Q$ frequency bins in the DFT domain, where the $Q \leq$ $L$ frequency bins may or may not be continuous. For each frequency bin $l_{q} \in \Phi_{l}, 0 \leq q \leq Q-1$, where $\Phi_{l}$ is the set of $Q$ frequency bin indexes, the same search grid of $K_{g}$ potential incident angles are used to generate matrices $\widetilde{\mathbf{A}}_{\mathbf{g}}^{\circ}[l]$ and $\widetilde{\mathbf{s}}_{\mathbf{g}}^{\circ}[l]$ as described in (11).

First, we construct two matrices: a block diagonal matrix $\widetilde{\mathbf{B}}_{\mathbf{g}}$ using $\widetilde{\mathbf{A}}_{\mathbf{g}}^{\circ}\left[l_{q}\right]$

$$
\widetilde{\mathbf{B}}_{\mathbf{g}}=\operatorname{blkdiag}\left\{\widetilde{\mathbf{A}}_{\mathbf{g}}^{\circ}\left[l_{1}\right], \widetilde{\mathbf{A}}_{\mathbf{g}}^{\circ}\left[l_{2}\right], \ldots, \widetilde{\mathbf{A}}_{\mathbf{g}}^{\circ}\left[l_{Q}\right]\right\}
$$

and a $\left(K_{g}+1\right) \times Q$ matrix $\mathbf{R}$ using $\widetilde{\mathbf{s}}_{\mathbf{g}}^{\circ}\left[l_{q}\right]$ with $\mathbf{R}=$ $\left[\widetilde{\mathbf{s}}_{\mathbf{g}}^{\circ}\left[l_{1}\right], \widetilde{\mathbf{s}}_{\mathbf{g}}^{\circ}\left[l_{2}\right], \ldots, \widetilde{\mathbf{s}}_{\mathbf{g}}^{\circ}\left[l_{Q}\right]\right]$. Then, we can obtain the following wideband virtual array model

$$
\widetilde{\mathbf{z}}=\widetilde{\mathbf{B}}_{\mathbf{g}} \widetilde{\mathbf{r}},
$$

where $\widetilde{\mathbf{z}}=\left[\mathbf{z}^{T}\left[l_{1}\right], \ldots, \mathbf{z}^{T}\left[l_{Q}\right]\right]^{T}$ and $\widetilde{\mathbf{r}}=\operatorname{vec}(\mathbf{R})$ is a $\left(K_{g}+\right.$ 1) $\cdot Q \times 1$ column vector by vectorizing $\mathbf{R}$.

We use the row vector $\mathbf{r}_{k}, 1 \leq k \leq K_{g}+1$, to represent $k$ th row of the matrix $\mathbf{R}$. Then, we form a new $\left(K_{g}+1\right) \times 1$ column vector $\hat{\mathbf{r}}$ based on the $l_{2}$ norm of $\mathbf{r}_{k}, 1 \leq k \leq K_{g}+1$, as given below

$$
\hat{\mathbf{r}}=\left[\left\|\mathbf{r}_{1}\right\|_{2},\left\|\mathbf{r}_{2}\right\|_{2}, \ldots,\left\|\mathbf{r}_{K_{g}+1}\right\|_{2}\right]^{T} .
$$

Finally, our group-sparsity based wideband DOA estimation method is formulated as follows

$$
\begin{aligned}
\min _{\tilde{\mathbf{r}}} & \|\hat{\mathbf{r}}\|_{1} \\
\text { subject to } & \left\|\widetilde{\mathbf{z}}-\widetilde{\mathbf{B}}_{\mathbf{g}} \widetilde{\mathbf{r}}\right\|_{2} \leq \varepsilon .
\end{aligned}
$$

The problem in (16) can be solved using CVX, a software package for specifying and solving convex programs [19], [20]. The first $K_{g}$ elements of the column vector $\hat{\mathbf{r}}$ are the wideband DOA estimation results over $K_{g}$ search grids.

\section{Simulation Results}

Consider an example with $M=4$ and $N=5$. With the sampling frequency twice the highest frequency of interest, the normalized frequencies of the impinging signals cover the range from $0.5 \pi$ to $\pi$, and the unit spacing $d=\lambda_{\min } / 2$. The number of signal samples in the time domain at each sensor is 32768 , and DFT of $L=64$ points is applied. Then, the number of data blocks used for estimating $\mathbf{R}_{\mathbf{x x}}[l]$ in (8) at each frequency bin is $P=512$. A search grid of $K_{g}$ incident angles is formed within the full angle range with a step size of $0.02^{\circ}$. The normalized frequency range of impinging signals covers the frequency bin set $\Phi_{l}=\{17,18, \cdots, 31\}$ with $Q=15$.

For the first set of simulations, there are 14 uncorrelated wideband signals impinging on the array, with incident angles uniformly distributed between $-70^{\circ}$ and $70^{\circ}$. The input SNR is $0 \mathrm{~dB}$ and the allowable error bound $\varepsilon$ is 10 for the narrowband method in (12) and 50 for our proposed wideband method in (16), which are chosen to give the best result for each method. The much larger value for $\varepsilon$ in the wideband case is due to the norm operation based on $Q=15$ frequencies instead of one single frequency. For the narrowband method, the best result is achieved at the highest frequency bin $l=31$, as for lower frequencies the relative spacing compared to 


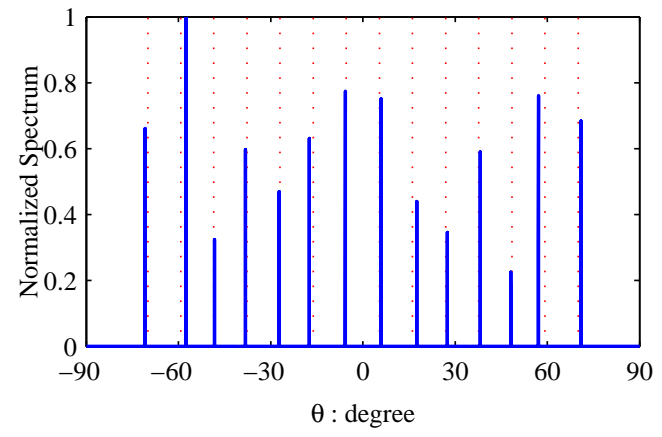

(a) Narrowband DOA estimation results.

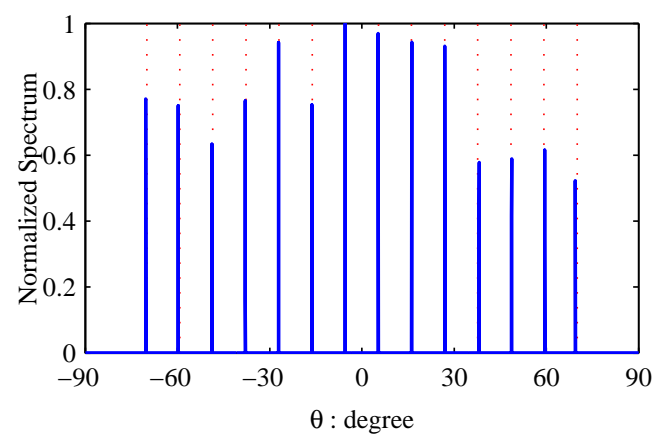

(b) Wideband DOA estimation results.

Fig. 2. (a) DOA estimation results obtained by the narrowband method for the frequency bin $l=31$. (b) DOA estimation results obtained by the wideband method. The dotted lines represent the actual incident angles of the impinging signals, while the solid lines represent the estimation results.

signal wavelength becomes shorter, leading to a relatively smaller array aperture. The results are shown in Fig. 2, where Fig. 2(a) gives the DOA estimation result obtained by the narrowband method, while Fig. 2(b) by the wideband method. It is clear that all 14 sources have been distinguished correctly by both methods, but the wideband method yields smaller estimation errors.

To clearly compare the estimation accuracy of the two methods with respect to a varied input SNR, the root mean square error (RMSE) results are shown in Fig. 3, where each point is based on an average of the results obtained by 500 simulation runs. It clearly shows that the proposed wideband method consistently outperforms the narrowband one with a big margin.

Finally, we give an example where the narrowband method clearly fails while our proposed method can still give a good result. The setting is the same as in the first set of simulations except that now the 14 source signals are uniformly distributed between $-60^{\circ}$ and $60^{\circ}$. The results are shown in Fig. 4, which again verifies the superior performance of the wideband method.

\section{CONCLUSiON}

A method for wideband DOA estimation with co-prime arrays has been proposed, where the received array signals are

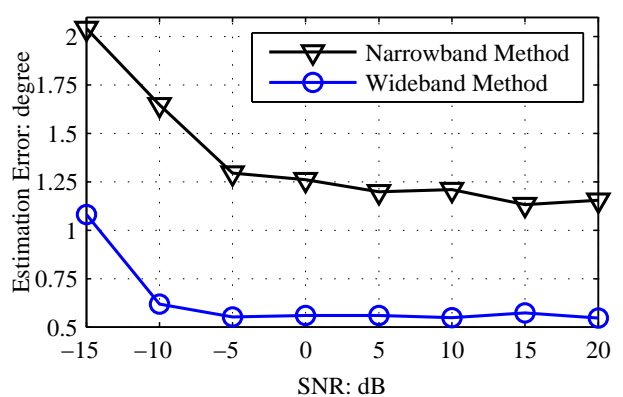

Fig. 3. Root mean square estimation error versus input SNR.

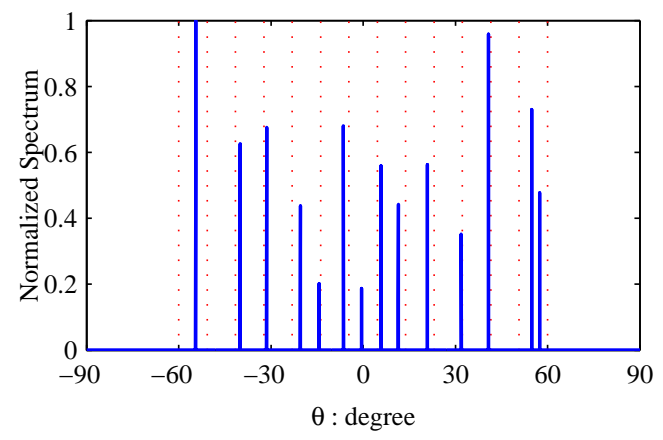

(a) Narrowband DOA estimation results.

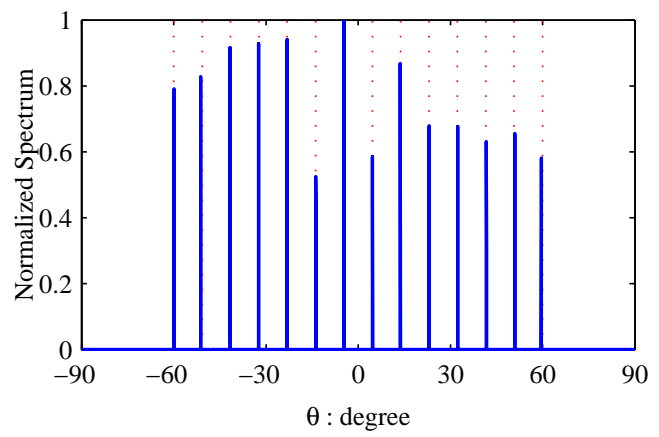

(b) Wideband DOA estimation results.

Fig. 4. (a) DOA estimation results obtained by the narrowband method for the frequency bin $l=31$. (b) DOA estimation results obtained by the wideband method. The dotted lines represent the actual incident angles of the impinging signals, while the solid lines represent the estimation results.

first partitioned into multiple frequencies/subbands and then a virtual array with a much larger aperture based on the coprime array concept is formed at each frequency/subband. By simultaneously exploiting the information at different frequencies/subbands, a group-sparsity based optimisation problem is formulated which is amenable to application of existing convex optimisation toolboxes. It has been shown by simulations that our proposed method can work effectively over a wide input SNR range and achieve a much better estimation result than the existing method using one frequency only. 


\section{REFERENCES}

[1] H. L. Van Trees, Optimum Array Processing, Part IV of Detection, Estimation, and Modulation Theory, Wiley, New York, 2002.

[2] W. Liu and S. Weiss, Wideband Beamforming: Concepts and Techniques, John Wiley \& Sons, Chichester, UK, 2010.

[3] Ralph T Hoctor and Saleem A Kassam, "The unifying role of the coarray in aperture synthesis for coherent and incoherent imaging," Proc. IEEE, vol. 78, no. 4, pp. 735-752, Apr. 1990.

[4] M. B. Hawes and W. Liu, "Location optimisation of robust sparse antenna arrays with physical size constraint," IEEE Antennas and Wireless Propagation Letters, vol. 11, pp. 1303-1306, November 2012.

[5] M. B. Hawes and W. Liu, "Sparse microphone array design for wideband beamforming," in Proc. International Conference on Digital Signal Processing, Santorini, Greece, July 2013.

[6] M. B. Hawes and W. Liu, "Compressive sensing based approach to the design of linear robust sparse antenna arrays with physical size constraint," IET Microwaves, Antennas \& Propagation, DOI: 10.1049/iet-map.2013.0469, 2014.

[7] Alan Moffet, "Minimum-redundancy linear arrays," IEEE Trans. Antennas Propag., vol. 16, no. 2, pp. 172-175, Mar. 1968.

[8] Palghat P Vaidyanathan and Piya Pal, "Sparse sensing with co-prime samplers and arrays," IEEE Trans. Signal Process., vol. 59, no. 2, pp. 573-586, Feb. 2011.

[9] Piya Pal and Palghat P Vaidyanathan, "Coprime sampling and the MUSIC algorithm,' in Proc. IEEE Digital Signal Processing Workshop and IEEE Signal Processing Education Workshop (DSP/SPE), Sedona, AZ, Jan. 2011, pp. 289-294.

[10] Piya Pal and Palghat P Vaidyanathan, "On application of LASSO for sparse support recovery with imperfect correlation awareness," in Proc. Asilomar Conference on Signals, Systems and Computers (ASILOMAR), Pacific Grove, CA, Nov. 2012, pp. 958-962.

[11] Yimin D Zhang, Moeness G Amin, and Braham Himed, "Sparsity-based DOA estimation using co-prime arrays," in Proc. IEEE International Conference on Acoustics, Speech and Signal Processing (ICASSP), Vancouver, Canada, May 2013, pp. 3967-3971.

[12] Yimin D Zhang, Moeness G Amin, Fauzia Ahmad, and Braham Himed, "DOA estimation using a sparse uniform linear array with two CW signals of co-prime frequencies," in Proc. IEEE International Workshop on Computational Advances in Multi-Sensor Adaptive Processing, Saint Martin, Dec. 2013, pp. 404-407.

[13] Guaning Su and Martin Morf, "The signal subspace approach for multiple wide-band emitter location," IEEE Trans. Acoust., Speech, Signal Process., vol. 31, no. 6, pp. 1502-1522, Dec. 1983.

[14] H Wang and M Kaveh, "Coherent signal-subspace processing for the detection and estimation of angles of arrival of multiple wide-band sources," IEEE Trans. Acoust., Speech, Signal Process., vol. 33, no. 4, pp. 823-831, Aug. 1985.

[15] Yeo-Sun Yoon, Lance M Kaplan, and James H McClellan, “TOPS: new DOA estimator for wideband signals," IEEE Trans. Signal Process., vol. 54, no. 6, pp. 1977-1989, June 2006.

[16] Zhang-Meng Liu, Zhi-Tao Huang, and Yi-Yu Zhou, "Direction-ofarrival estimation of wideband signals via covariance matrix sparse representation," IEEE Trans. Signal Process., vol. 59, no. 9, pp. 42564270, Sept. 2011.

[17] Zhang-Meng Liu, Zhi-Tao Huang, and Yi-Yu Zhou, "Sparsity-inducing direction finding for narrowband and wideband signals based on array covariance vectors," IEEE Trans. Wireless Commun., vol. 12, no. 8, pp. 3896-3906, Aug. 2013.

[18] Ji-An Luo, Xiao-Ping Zhang, and Zhi Wang, "A new subband information fusion method for wideband DOA estimation using sparse signal representation," in Proc. IEEE International Conference on Acoustics, Speech and Signal Processing (ICASSP), Vancouver, Canada, May 2013, pp. 4016-4020.

[19] Michael Grant and Stephen Boyd, "CVX: Matlab software for disciplined convex programming, version 2.0 beta, build 1023,” Dec. 2013.

[20] M. Grant and S. Boyd, "Graph implementations for nonsmooth convex programs," in Recent Advances in Learning and Control, V. Blondel, S. Boyd, and H. Kimura, Eds., Lecture Notes in Control and Information Sciences, pp. 95-110. Springer-Verlag Limited, 2008, http://stanford. edu/ boyd/graph_dcp.html. 